\title{
Considérations géométriques, destinées à faciliter l'étude de la théorie des transcendantes elliptiques.
}

(Par M. C. Küpper à Trèves.)

1. Lemme. Torsque le côté $A B$ d'un triangle $A B C$ tourne infiniment peu sur un de ses points $O$, les deux autres côtés éprouvent des variations $d C A, d C B$, telles, que:

$$
\frac{d C A}{d C B}=\frac{C A}{C B} \cdot \frac{O A}{O B} .
$$

C'est la relation connue entre les six segments, qu'une transversale détermine sur les côtés d'un triangle.

Remarque. A l'aide de ce lemme on peut intégrer géométriquement certaines équations différentielles:

$1^{\text {er }}$ exemple. Si $O$ est situé sur la bissectrice de l'angle $A C B$, on a $\frac{O A}{O B}=\frac{C A}{C B}$, donc, en introduisant les variables $y=C A, x=C B$, en vertu du lemme:

$$
\frac{d x}{x^{2}}+\frac{d y}{y^{2}}=0
$$

L'intégration fournit $\frac{1}{x}+\frac{1}{y}=$ une constante. D'autre part, puisque les droites $A B$ passent par $O$, cette équation doit avoir lieu.

$2^{\text {e }}$ exemple. Si $A B$ touche constamment une hyperbole, dont $C A$, $C B$ sont les asymptotes, le point de contact $O$ divisera $A B$ en deux parties égales, par conséquent:

$$
\frac{d x}{x}+\frac{d y}{y}=0 \text {. }
$$

En intégrant on trouve $x y=$ une constante, ce qui résulte également des propriétés de l'hyperbole.

On pourrait multiplier ces exemples; nous nous contentons d'en étudier celui, auquel se rattachent quelques conséquences importantes.

2. Thé or ème. Étant données deux coniques homofocales et de même espèce $E, E^{\prime}$; si par un point pris arbitrairement sur $E^{\prime}$ on mène deux tangentes $\grave{a} E$, l'excès de la somme de ces tangentes sur l'arc de $E$, compris entre les points de contact est une quantité constante. 
Par les extrémités $P, P^{\prime}$ d'un élément de $E^{\prime}$ soient menées à $E$ les quatre tangentes $P T_{1}, P T_{2}, P^{\prime} T_{1}^{\prime}, P^{\prime} T_{2}^{\prime}$. Les droites $P T_{1}, P T_{2}$ étant également inclinées sur l'élément $P P^{\prime}$, la variation totale de la somme $P T_{1}+P T_{2}$ sera $-T_{1} T_{1}^{\prime}+T_{2} T_{2}^{\prime}$, ou égale à la différence des $\operatorname{arcs} T_{1} T_{2}, T_{1}^{\prime} T_{2}^{\prime}$; par suite:

$$
P T_{1}+P T_{2}-\operatorname{arc} T_{1} T_{2}=\text { une constante. }
$$

En d'autres termes: Lorsque les angles, circonscrits à deux arcs d'une conique $\boldsymbol{E}$ ont leurs sommets sur une conique $E^{\prime}$, homofocale à $E$, la différence de ces arcs peut être construite, ou exprimée par une fonction algébrique des coordonnées de leurs extrémitès. Afin d'abréger, en parlant de coniques homofocales, nous supprimons l'expression "de même espèce", bien qu'elle soit toujours sous - entendue.

3. Thè or èm e. Lorsque le point de concours de deux tangentes d'une conique $E$ décrit l'élément d'une conique homofocale $E^{\prime}$, les déplacements infiniment petits des points de contact sont entre eux comme les carrés des tangentes.

En conservant la notation précédente, il s'agit d'exprimer le rapport $T_{1} T_{1}^{\prime}: T_{2} T_{2}^{\prime}$ ou $d s_{1}: d s_{2}$. Pendant que $P$ se meut sur la droite $P P^{\prime}$, la corde de contact $T_{1} T_{2}$ glisse sur le point $O$, pôle de $P P^{\prime}$. Les quatre droites $P T_{1}$, $P O, P T_{2}, P P^{\prime}$ forment un faisceau harmonique, et parceque $P P^{\prime}$ est la bissectrice du supplément de l'angle $T_{1} P T_{2}, P O$ sera celle de $T_{1} P T_{2}$; donc $\frac{O T_{1}}{O T_{2}}=\frac{P T_{1}}{P T_{2}} ;$ ensuite, d'après 1 .

$$
\frac{d s_{1}}{d s_{2}}=\frac{P T_{1}^{2}}{P T_{2}^{2}}
$$

Remarque. Si $E, E^{\prime}$ sont deux ellipses, le rapport $P T_{1}: P T_{2}$ peut ètre remplacé par le rapport des diamètres de $E$, parallèles à ces tangentes; cela se voit immédiatement quand on considère $\boldsymbol{E}$ comme la projection orthogonale d'un cercle, qui a pour diamètre le grand axe de $E$. Dans ce cas, pour déterminer un point $T$ ou $(x, y)$ de $E$, nous nous servons de l'angle $\psi$, au moyen duquel $x, y$ s'expriment par $a \sin \psi, b \cos \psi,(a, b$ étant les demiaxes de $E)$; et nous appellerons un point, ainsi déterminé par l'angle $\psi$, simplement le point $\psi$. Maintenant, en exprimant $d s_{1}, d s_{2}$ et les diamètres, qui leur sont parallèles en fonctions de $\psi_{1}, \psi_{2}$, ce qui se fera encore à l'aide de propriétés projectives, on est conduit à l'équation différentielle:

$$
\text { (I.) } \frac{d \psi_{1}}{\Delta \psi_{1}}=\frac{d \psi_{2}}{\Delta \psi_{2}}, \quad k^{2}=\frac{a^{2}-b^{2}}{a^{2}} \text {. }
$$

Journal für Mathematik Bd. LXIII. Heft 1. 
Pour le cas de deux hyperboles, on établira sans difficulté une équation analogue, mais les angles, qu'il faudrait introduire, n'ayant pas une signification géométrique assez simple, nous resterons dans l'hypothèse de deux ellipses.

Lorsqu'il s'agit de deux paraboles $y^{2}=2 p x, y^{2}=2 q x$, on trouve:

$$
\frac{d^{3} y_{1}}{\sqrt{1+\left(\frac{y_{1}}{p}\right)^{2}}}=\frac{d y_{2}}{\sqrt{1+\left(\frac{y_{2}}{p}\right)^{2}}}
$$

( $y_{1}, y_{2}$ sont les coordonnées de deux points $T_{1}, T_{2}$ de la première parabole). En opérant sur cette équation, comme nous allons le faire sur (I.), on en déduira les propriétés fondamentales des fonctions exponentielles.

4. Addition des fonctions elliptiques. Pour plus de brièveté nous dirons, que deux arcs de conique $E$ sont égaux, lorsqu'ils sont compris entre les côtés de deux angles, circonscrits à cette conique, et dont les sommets sont situés sur une conique homofocale $E^{\prime}$. Bien que ces arcs aient une différence généralement différente de zéro, qu'on ne manquera pas d'appercevoir, quand on représente les arcs comme il a été fait dans le $n^{\circ} 2$, et précisément à cause de cette dernière circonstance l'expression adoptée ne peut donner lieu à une équivoque.

En intégrant les deux membres de l'équation (I.) entre les limites correspondantes $0, \psi_{1}$ et $\psi_{0}, \psi_{2}$, il vient:

$$
\begin{gathered}
\int_{11}^{\psi_{1}} \frac{d \psi_{1}}{\Delta \psi_{1}}=\int_{\psi_{0}}^{\psi_{2}} \frac{d \psi_{2}}{\Delta \psi_{2}}, \text { ou, en posant } \int_{0}^{\psi} \frac{d \psi}{\Delta \psi}=F(k, \psi)=u, \psi=\operatorname{am} u, \\
\text { (II.) } u_{0}+u_{1}=u_{2} .
\end{gathered}
$$

Il en résulte cet énoncé: Si l'on prend pour limites d'intégrales elliptiques de première espèce les angles, qui déterminent les extrémités d'arcs d'ellipse égaux, ou en un mot les limites de tels arcs, ces intégrales auront la même valeur. Ainsi, l'égalité d'arcs d'ellipse, telle que nous l'avons définie, consiste en ce que les intégrales de $1^{\text {ère }}$ espèce, qui ont les mêmes limites que ces arcs sont égales; tandis que les intégrales correspondantes de $2^{e}$ espèce ont des différences algébriques.

Pour intégrer géométriquement l'équation (I.), on n'a donc qu'a construire un arc d'ellipse égal à un arc donné, ce qui se fera d'après le $n^{\circ} 2$. Soient par exemple $\psi_{1}, \psi_{2}$ les limites de l'arc donné, $\psi_{3}, \psi_{4}$ celles d'un arc qui lui est égal, les arcs, limités par $\psi_{1}, \psi_{3}$ et $\psi_{2}, \psi_{4}$ étant encore égaux, 
on a ce théorème: Quand deux sommets opposés d'un quadrilatère circonscrit à une conique sont situés sur une conique homofocale, les côtés opposés de ce quadrilatère se coupent sur une seconde conique homofocale.

La solution géométrique du problème de l'addition se trouve suffisamment indiquée. Si dans l'équation (II.) on attribue à $\psi_{0}$ une valeur déterminée, on obtient tous les couples de valeurs correspondantes $\psi_{1}, \psi_{2}$ à l'aide d'une certaine ellipse $E^{\prime}$, homofocale à $E$. Parceque $E^{\prime}$ doit contenir le point d'intersection des deux tangentes à $E$ aux points 0 et $\psi_{v}$, elle est complètement déterminée; on l'appellera la conjuguée de $\boldsymbol{E}$ par rapport à l'angle $\psi_{0}$ ou à am $u_{0}$. Réciproquement, deux ellipses homofocales $E, E^{\prime}$ étant données, on saura toujours assigner l'angle $\psi_{11}$, par rapport auquel l'une $E^{\prime}$ sera la conjuguée de $E$.

On a pour les demi-axes $\sqrt{a^{2}+\lambda_{0}}, \sqrt{b^{2}+\lambda_{0}}$ de $E^{\prime}$ :

$$
\text { (III.) }\left\{\begin{array}{cc}
\sqrt{a^{2}+\lambda_{0}}=a \sqrt{\frac{1+\Delta \psi_{0}}{1+\cos \psi_{0}}}, & \sqrt{b^{2}+\lambda_{0}}=b \sqrt{\frac{1+\Delta \psi_{0}}{\cos \psi_{0}+\Delta \psi_{0}}} \\
\text { Et: } \cos \psi_{0}=\frac{a^{2} b^{2}-\lambda_{0}^{2}}{a^{2} b^{2}+2 a^{2} \lambda_{0}+\lambda_{0}^{2}}, & \sin \psi_{0}=\frac{2 a \sqrt{\left(a^{2}+\lambda_{0}\right)\left(b^{2}+\lambda_{0}\right) \lambda_{0}}}{a^{2}\left(b^{2}+\lambda_{0}\right)+\lambda_{0}\left(a^{2}+\lambda_{0}\right)} \\
\Delta \psi_{0}=\frac{b^{2}\left(a^{2}+\lambda_{0}\right)+\lambda_{0}\left(b^{2}+\lambda_{0}\right)}{a^{2}\left(b^{2}+\lambda_{0}\right)+\lambda_{0}\left(a^{2}+\lambda_{0}\right)}
\end{array}\right.
$$

En tenant compte de la relation géométrique, qui existe entre les points $0, \psi_{0}$ et $\psi_{1}, \psi_{2}$; savoir, que les tangentes en ces points se coupent sur $E^{\prime}$, homofocale à $E$, on déduit aisément:

$$
\cos \psi_{0}=\cos \psi_{1} \cos \psi_{2}+\sin \psi_{1} \sin \psi_{2} \Delta \psi_{10} .
$$

Quant à la différence des arcs d'ellipse, limités par $0, \psi_{10}$ et $\psi_{1}, \psi_{2}$, on l'évaluera de la manière suivante:

Sơient $\boldsymbol{T}_{1} \boldsymbol{P}, \boldsymbol{T}_{2} \boldsymbol{P}$ les tangentes en $\psi_{1}, \psi_{2}$. Si l'on considère $\boldsymbol{E}$ comme la projection orthogonale du cercle, décrit sur $2 a$ comme diamètre, on reconnait sur le champ que

$$
T_{1} P=a \operatorname{tang} \frac{1}{2}\left(\psi_{2}-\psi_{1}\right) \Delta \psi_{1}, \quad T_{2} P=a \operatorname{tang} \frac{1}{2}\left(\psi_{2}-\psi_{1}\right) \Delta \psi_{2} .
$$

En écrivant $\frac{1-\cos \left(\psi_{2}-\psi_{1}\right)}{\sin \left(\psi_{2}-\psi_{1}\right)}$ au lieu de $\operatorname{tang} \frac{1}{2}\left(\psi_{2}-\psi_{1}\right)$, et faisant usage des formules:

$$
\begin{array}{r}
\sin \psi_{0} \Delta \psi_{1}=\cos \psi_{1} \sin \psi_{2}-\sin \psi_{1} \cos \psi_{2} \Delta \psi_{0} \\
\sin \psi_{0} \Delta \psi_{2}=\cos \psi_{1} \sin \psi_{2} \Delta \psi_{0}-\sin \psi_{1} \cos \psi_{2} \\
6^{*}
\end{array}
$$


on obtient:

$$
T_{1} P+T_{2} P=a \frac{1-\cos \psi_{0}}{\sin \psi_{0}}\left(1+\Delta \psi_{0}\right)+\frac{\cos \psi_{0}-\cos \left(\psi_{2}-\psi_{1}\right)}{\sin \psi_{0}}\left(1+\Delta \psi_{0}\right),
$$

équation qui à l'aide de la formule

$$
\cos \psi_{0}=\cos \psi_{1} \cos \psi_{2}+\sin \psi_{1} \sin \psi_{2} \Delta \psi_{0}
$$

se change en

$$
T_{1} P+T_{2} P=a \operatorname{tang} \frac{1}{2} \psi_{0}\left(1+\Delta \psi_{10}\right)-a k^{2} \sin \psi_{1} \sin \psi_{1} \sin \psi_{2} .
$$

Posant $\psi_{1}=0, \psi_{2}=\psi_{0}$, cette somme devient: $a \operatorname{tang} \frac{1}{2} \psi_{0}\left(1+\Delta \psi_{0}\right)$. Telle est donc la somme des tangentes en $0, \psi_{0}$; par conséquent il vient pour la différence des $\operatorname{arcs}$ en question: $a k^{2} \sin \psi_{0} \sin \psi_{1} \sin \psi_{2}$.

5. Multiplication. Soit toujours $E^{\prime}$ la conjuguée de $E$ par rapport à l'angle $\psi_{0}$. Tirons dans $E^{\prime}$ des cordes consécutives, de manière qu'ellés touchent $\boldsymbol{E}$ aux points $\psi_{1}, \psi_{2}, \ldots \psi_{n+1}$, nous aurons:

$$
\boldsymbol{F}\left(k, \psi_{n+1}\right)=n \boldsymbol{F}\left(k, \psi_{0}\right)+\boldsymbol{F}\left(k, \psi_{1}\right), \quad \text { où } \psi_{1} \text { est quelconque, p. e. }=\mathbf{0} .
$$

Donc, $\psi_{0}=\operatorname{am} u_{0}$ étant donnée, on saura construire l'amplitude d'une intégrale, égale à un multiple $n$ de $u_{0}$.

Lorsqu'en procédant de cette sorte, il arrive que la $n+1^{\text {ième }}$ corde s'applique sur la première, $u_{0}$ et $4 F\left(k, \frac{\pi}{2}\right)=\omega$ seront commensurables; et réciproquement, pour qu'une corde ultérieure coincide avec la première, il est nécessaire et il suffit, qu'on ait $u_{0}=\frac{h}{n} \omega, h$ étant premier avec $n$. Supposons, que l'angle $\psi_{0}=\operatorname{am} u_{1}$ satisfasse à cette condition: Alors de ce qu'elle est indépendante de l'angle $\psi_{1}$, qui marque sur $E$ le point de contact de la première corde, on conclura qu'il existe une infinité de polygones circonscrits à $E$ et inscrits à $E^{\prime}$, de sorte, que telle tangente à $E$, qu'on voudra, puisse devenir le côté de l'un d'entre eux. Tous ces polygones ont $n$ côtés; leurs périmètres feront un nombre constant de fois $h$ le tour entier de l'espar,e angulaire, c'est-à-dire qu'ils sont tous circonscrits à un multiple constant $h$ de l'ellipse entière $E$, et inscrits à ce même multiple de $E^{\prime}$. En outre on découvre aisément, qu'ils jouissent des propriétés suivantes:

I. Les polygones, qui sont à la fois circonscrits à une ellipse $E$, et inscrits à une ellipse homofocále $E^{\prime}$ ont des périmètres égaux.

Ce périmètre peut être évalué par les formules équivalentes:

$$
\text { (a.) } \quad 2 \sqrt{\left(a^{2}+\lambda_{10}\right)\left(b^{2}+\lambda_{1}\right) \lambda_{0}} \Sigma \frac{(\Delta \psi)^{2}}{b^{2}+\lambda_{0}(\Delta \psi)^{2}},
$$


la somme renfermant tous les angles $\psi_{1}, \psi_{2}, \ldots$,

$$
\text { ( } \beta \text {.) } 2 \sqrt{\left(a^{2}+\lambda_{0}\right)\left(b^{2}+\lambda_{0}\right) \lambda_{0}} \Sigma \frac{1}{\lambda_{0}+d^{2}},
$$

où $d$ représente un demi-diamètre de $E$ parallèle à un côté du polygone, le signe somme s'étendant à tous ces côtés,

$$
\text { ( } \gamma \text {. }) \quad \sqrt{\left.\left(a^{2}+\lambda_{0}\right)\left(b^{2}+\lambda_{0}\right) \lambda_{1}\right)} \Sigma \frac{\left(a^{2}+\lambda_{0}\right)+\left(b^{2}+\lambda_{0}\right)-n^{2}}{\left(a^{2}+\lambda_{0}\right)\left(b^{2}+\lambda_{0}\right)-\lambda_{0} n^{2}},
$$

où $n$ représente les distances $n_{1}, n_{2}, \ldots$ du centre de $E$ aux différentes normales qu'on auraìt menées à $E$ en $\psi_{11}, \psi_{1}, \ldots$

II. Entre tous les polygones convexes $p_{h}$ de $n$ côtès et dont les périmòtres sont inscrits au même multiple $h$ de l'ellipse entière $E^{\prime}$, ceux qui sont circonscrits à une ellipse homofocale $E$, ont un périmètre maximum.

III. Entre tous les polygones convexes $p_{h}$ de $n$ côtés et dont les périmètres se trouvent circonscrits au même multiple $h$ de l'ellipse entière $E$, ceux qui sont inscrits à une ellipse homofocale $E_{1}$ ont un périmètre minimum.

Les numéros II., III. contiennent la solution d'une question soulevée par M. Steiner (Journal de Liouville, avril 1841). Avant d'aborder le $\mathbf{n}^{\circ}$ III., on résoudra ce problème:

Étant donnés un arc de conique et langle circonscrit; tirer à cet arc une tangente de telle manière, que la ligne brisée convexe, qui joint les extrémités de l'arc donné et qui se compose de deux parties des tangentes extrêmes et du segment intercepté par ces dernières sur la tangente intermédiaire, soit la plus petite possible.

On verra, que la tangente cherchée doit toucher l'arc à son milieu, ou, on trouvera ses points d'intersection avec les tangentes extrêmes sur une conique homofocale à celle, dont l'arc donné fait partie.

Quant à la construction, voyez $\mathrm{n}^{\circ} 6$.

IV. L'ellipse $E^{\prime}$ qui contient les sommets d'un polygone $p_{h}$ est la conjuguée de $E$ par rapport à l'angle am $\frac{h}{n} \omega$, les points de contact $\psi_{1}, \psi_{2}, \ldots$ des côtés de $p_{h}$ avec $E$ sont séparés par des arcs d'ellipse égaux, auquels correspondent des intégrales de première espèce, ayant toutes la même valeur: $\frac{h}{n} \omega$. On pourra ranger les points $\psi$ dans un autre ordre, de manière qu'ils soient encore séparés par un intervalle constant, et que l'intégrale correspon- 
dante soit un multiple quelconque de $\frac{h}{n} \omega$. Désignons par $\frac{q h}{n} \omega$ ce multiple, et supposons $q$ premier avec $n$ : alors il est clair qu'on passera par tous les points $\psi$, avant de revenir au point de départ $\psi_{1}$. Si donc, dans cet arrangement, on remplace les points $\psi$ par les côtés correspondants de $p_{h}$, en prolongeant chacun d'eux jusqu'à la rencontre du suivant, on formera de nouveaux polygones de $n$ côtés, circonscrits à $E$ et inscrits dans des ellipses homofocales à $E$. Les angles, par rapport auquels ces dernières seront les conjuguées de $E$, se trouvent compris dans l'expression am $\frac{\nu}{n} \omega$, où l'on entend par $\nu$ un nombre premier avec $n$, et plus petit que $\frac{1}{2} n$; et il $\mathrm{y}$. aura autant de ces ellipses, qu'il y a des nombres $\nu$, savoir $\frac{1}{2} \varphi(n)$.

Les mêmes angles am $\frac{\nu}{n} \omega$ permettent de distinguer entre eux les polygones circonscrits à $E$, en tant qu'ils sont inscrits à l'une ou l'autre des $\frac{1}{2} \varphi(n)$ ellipses homofocales; car le nombre $\nu$ indique, combien de fois le périmètre du polygone correspondant fait le tour entier de l'espace angulaire, ou à quel multiple de $E$ il est circonscrit. En supposant $\nu=1$, on se voit conduit à des polygones $p_{1}$, dont les côtés donnent par leurs points de contact avec $E$, la division de cette courbe en $n$ parties égales; ainsi on peut toujours ranger les points $\psi$ dans un tel ordre, qu'ils forment les points de cette division.

Actuellement, lorsqu'il s'agit d'exécuter cette division, on y employera l'une des $\frac{1}{2} \varphi(n)$ ellipses, et il faudra déterminer ses demi-axes $\sqrt{a^{2}+\lambda_{1}}$, $\sqrt{b^{2}+\lambda_{0}}$. A cette fin on peut avoir recours aux formules relatives à la division des fonctions elliptiques; mais on parvient à établir une équation entre $\cos$ am $\frac{\omega}{n}$, et $\Delta \mathrm{am} \frac{\omega}{n}$ à l'aide de certaines considérations géométriques, qui découlent des constructions mêmes, dont nous nous occupons. Cette équation une fois formée, on y remplacera $\cos a m \frac{\omega}{n}, \Delta \mathrm{am} \frac{\omega}{n}$ par leurs valeurs en $\lambda_{0}$ (voir $\mathrm{n}^{\circ} 4$ ).

Remarque. Par voie de dualité on trouve les polygones inscrits dont les côtés sous - tendent des arcs d'ellipse égaux, et qui sont circonscrits aux polaires réciproques d'ellipses homofocales à $E$; mais nous croyons inutile d'insister là-dessus.

6. Problème. Partager en deux parties égales un arc d'ellipse $E$ (ou de conique), ou trouver le milieu $M$ d'un tel arc. 
Aux extrémités $T_{1}, T_{2}$ de l'arc donné (Fig. 1) tirons les tangentes $T_{1} P, T_{2} P$, et par leur point de concours $P$ faisons passer une ellipse (conique) $E^{\prime}$ homofocale à $E$. Par un sommet $B_{1}$ de $E^{\prime}$ menons deux tangentes à $E$; elles comprendront entre elles un arc d'ellipse, égal à $T_{1} T_{2}$, et dont le milieu est le sommet $B$ de $E$. Par les deux points, où la tangente en $B$ rencontré les deux tangentes qui se coupent en $B_{1}$ on fera passer une ellipse (conique) $E^{\prime \prime}$ homofocale à $E$. Alors, en joignant les points d'intersection de $E^{\prime \prime}$ avec les tangentes $T_{1} P$, $T_{2} P$, savoir les points $X_{1}, X_{2}$ on aura la

Fig. 1.

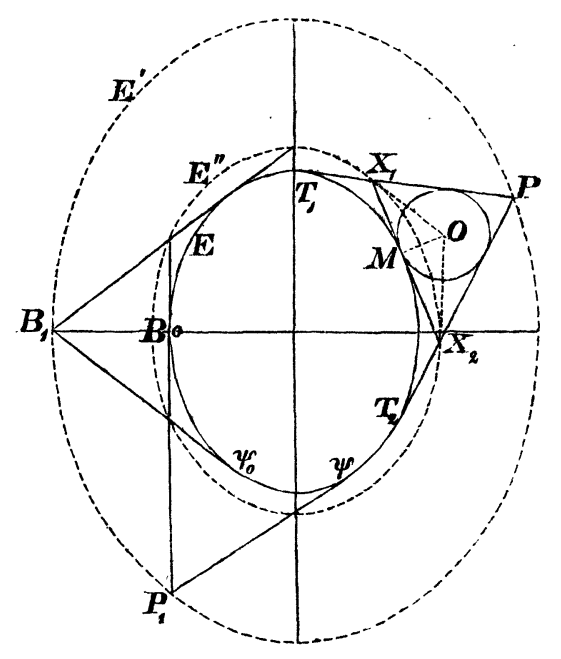
tangente au milieu cherché $M$.

On sait que les tangentes $X_{1} O, X_{2} O$ à $E^{\prime \prime}$ sont les bissectrices des angles $P X_{1} X_{2}, P X_{2} X_{1}$; donc $O$ est le centre de la circonférence inscrite au triangle $P X_{1} X_{2}$. Or, parceque $X_{1} X_{2}$, polaire de $O$ par rapport à $E^{\prime \prime}$ est tangente à l'ellipse (conique) homofocale $E, O M$ doit être perpendiculaire à $X_{1} X_{2}$. De là on voit, que la circonférence inscrite à $P X_{1} X_{2}$ touche la droite $X_{1} X_{2}$ en $M$; ou bien l'arc $T_{1} T_{2}$ en son milieu.

Ensuite, en exprimant la différence des arcs $T_{1} M, M T_{2}$ d'après le $n^{\circ} 2$; on la trouve $=M X_{2}+X_{2} T_{2}-M X_{1}-X_{1} T_{1}=T_{2} P-T_{1} P$; d'où suit le

Théorème. Lorsqu'à un triangle mixtiligne, formé par deux tangentes d'une conique et l'arc compris entre elles, une circonférence est inscrite, le point de contact de la circonférence avec la conique partage l'arc de celle-ci en deux parties, dont la différence est égale à la différence des deux tangentes.

Depuis la publication des ,producioni matematiche" de Fagnano on savait partager le quart d'ellipse en deux parties, dont la différence égale celle des demi-axes; mais le procédé qui résulte des recherches du géomètre italien ne fait entrevoir ni le théorème général, ni la construction que ce théorème fournit pour le cas particulier d'un quart d'ellipse.

Quand l'une des extrémités de l'arc à diviser est un sommet de $E$, la construction se simplifie encore: Prenons par exemple l'arc limité par les 
points o, (sommet du petit axe de $E$ ) et $\psi$; les tangentes en ces points se coupant en $P_{1}$, on fera passer par $P_{1}$ la conjuguée de $E$ par rapport à l'angle $\psi$. Par le sommet $B_{1}$ de cette ellipse on tirera une tangente à $E$, le point de contact $\psi_{0}$ sera le milieu cherché. En employant le même procédé, pour diviser en deux parties égales l'arc $o \psi_{10}$, on obtiendra la sous-division de l'arc donné en $2^{2}$ parties égales, et ainsi de suite.

Sans doute on ne doit pas attribuer une valeur pratique à cette méthode géométrique de diviser en $2^{n}$ parties égales une intégrale de première espèce; mais elle nous parait digne d'être remarquée par la raison qu'elle présente à l'esprit une image nette et précise de cette division.

7. En soumettant la construction précédente à l'analyse, on est assez naturellement conduit à quelques formules fondamentales concernant la multiplication des fonctions elliptiques.

Conformément au théorème, que nous venons d'énoncer, la différence $\delta$ des deux $\operatorname{arcs} \boldsymbol{T}_{1} \boldsymbol{M}, \boldsymbol{M} \boldsymbol{T}_{2}$ dont les extrémités correspondent aux points $\psi_{1}$, $\mu$ et $\mu, \psi_{2}$ est:

$$
\delta=a \operatorname{tang} \frac{1}{2}\left(\psi_{2}-\psi_{1}\right)\left(\Delta \psi_{2}-\Delta \psi_{1}\right) .
$$

D'un autre côté $\left(n^{\circ} 4\right)$ on a

donc :

$$
\delta=a k^{2} \sin \mu \sin \psi_{0}\left(\sin \psi_{1}-\sin \psi_{2}\right),
$$

$$
\sin \mu=\frac{\cos \psi_{1}-\cos \psi_{2}}{\sin \psi_{0}\left(\Delta \psi_{1}+\Delta \psi_{2}\right)}
$$

Et comme $\mu$ se change en $\psi_{0}$, quand $\psi_{1}, \psi_{2}$ deviennent $o, \psi$, on aura:

$$
\sin ^{2} \psi_{0}=\frac{1-\cos \psi}{1+\Delta \psi} \quad \text { et } \cos ^{2} \psi_{0}=\frac{\cos \psi+\Delta \psi}{1+\Delta \psi}, \quad\left(\Delta \psi_{0}\right)^{2}=\frac{\cos \psi+\Delta \psi}{1+\cos \psi} .
$$

Posons $\cos \psi=\cos \operatorname{am} u=c, \frac{\cos \psi}{\Delta \psi}=\sin \operatorname{coam} u=s, \cos \psi_{0}=c_{i}, \frac{\cos \psi_{0}}{\Delta \psi_{0}}=s_{0}$, il vient:

$$
c_{0}^{2}=\frac{c(1+s)}{c+s}, \quad s_{0}^{2}=\frac{s(1+c)}{c+s}
$$

par conséquent:

$$
c=\frac{-1+c_{0}^{2}+s_{0}^{2}}{1-c_{0}^{2}+s_{0}^{2}}, \quad s=\frac{-1+c_{0}^{2}+s_{0}^{2}}{1+c_{0}^{2}-s_{0}^{2}}, \quad \frac{\sin \psi}{\sin \psi_{0}}=\frac{2 s}{1-c_{0}^{2}+s_{0}^{2}} .
$$

$O u, h$ désignant un nombre entier quelconque: 


$$
\begin{aligned}
& \text { (VI.) } \quad \operatorname{cosam} 2 h u_{0}=\frac{\sin ^{2} \operatorname{coam} h u_{0}-\sin ^{2} a m h u_{0}}{\sin ^{2} \operatorname{coam} h u_{0}+\sin ^{2} a m h u_{0}} \\
& \text { (VII.) } \quad \frac{\sin \operatorname{am} 2 h u_{0}}{s_{0} \sin a m u_{0}}=\frac{2 \frac{\sin \operatorname{am} h u}{\sin a m u} \cdot \frac{\sin \operatorname{coam} h u}{s_{0}}}{\sin ^{2} \operatorname{coam} h u+\sin ^{2} a m h u}
\end{aligned}
$$

Si dans l'expression générale de $\sin \mu$ on remplace $\Delta \psi_{1}+\Delta \psi_{2}$ par

$$
\frac{1+\Delta \psi}{\sin \psi} \sin \left(\psi_{2}-\psi_{1}\right) \quad\left(\text { voir } n^{\circ} 4 .\right)
$$

elle devient:

$$
\sin \mu=\frac{\cos \psi_{1}-\cos \psi_{2}}{\sin \psi_{0} \frac{1+\Delta \psi}{\sin \psi} \sin \left(\psi_{2}-\psi_{1}\right)}
$$

Les formules (V.) donnent: $\frac{1+\Delta \psi}{\sin \psi}=\frac{1}{s_{0} \sin \psi_{0}}$, donc

$$
\sin \mu=\frac{s_{0}\left(\cos \psi_{1}-\cos \psi_{2}\right)}{\sin \left(\psi_{2}-\psi_{1}\right)}
$$

Ensuite, de $\cos \psi_{0}=\cos \psi_{1} \cos \mu+\sin \psi_{1} \sin \mu \Delta \psi_{0}=\cos \psi_{2} \cos \mu+\sin \psi_{2} \sin \mu \Delta \psi_{0}$ on tire:

$$
\operatorname{tang} \mu=\frac{\cos \psi_{1}-\cos \psi_{2}}{\Delta \psi_{0}\left(\sin \psi_{2}-\sin \psi_{1}\right)}
$$

donc:

$$
\cos \mu=\frac{c_{0}\left(\sin \psi_{2}-\sin \psi_{1}\right)}{\sin \left(\psi_{2}-\psi_{1}\right)}
$$

En posant $\psi_{1}=\operatorname{am} 2 h u_{0}, \psi_{2}=\operatorname{am} 2(h+1) u_{1}$, et $\mu=\operatorname{am}(2 h+1) u_{1}$, on a :

(VIII.) $\frac{\sin \operatorname{am}(2 h+1) u_{0}}{\sin \operatorname{am} u_{0}}=\frac{\frac{\cos \operatorname{am} 2 h u_{0}}{\sin \operatorname{am} u_{0}}-\frac{\cos \operatorname{am} 2(h+1) u_{0}}{\sin \operatorname{am} u_{0}}}{\frac{\sin \operatorname{am} 2(h+1) u_{0}}{s_{0}} \cos \operatorname{am} 2 h u_{0}-\frac{\sin \operatorname{am} 2 h u_{0}}{s_{0}} \operatorname{cosam} 2(h+1) u_{0}}$,

(IX.) $\frac{\cos \operatorname{am}(2 h+1) u_{0}}{c_{0}}=\frac{\frac{\sin \operatorname{am} 2(h+1) u_{0}}{s_{0} \sin \operatorname{am} u_{0}}-\frac{\sin \operatorname{am} 2 h u_{0}}{s_{0} \sin \operatorname{am} u_{0}}}{\frac{\sin \operatorname{sm} 2(h+1) u_{0}}{s_{0} \sin \operatorname{sm} u_{0}} \cos \operatorname{am} 2 h u_{0}-\frac{\sin 2 h u_{0}}{s_{0} \sin \operatorname{am} u_{0}} \cos \operatorname{am} 2(h+1) u_{0}}$.

Mr. Richelot a fait remarquer, que les expressions en $c_{0}, s_{0}$, qui donnent $\frac{\sin a m n u_{0}}{\sin a m u_{0}}$ et $\cos a m n u_{0}$ se changent en $\frac{\cos \operatorname{coam} n u_{0}}{\cos \cos n u_{0}}$ et $\sin \operatorname{coam} n u_{0}$, par la permutation des lettres $c_{v}, s_{0}$.

Journal für Mathematik Bd. LXIII. Heft 1. 
Fig. 2.

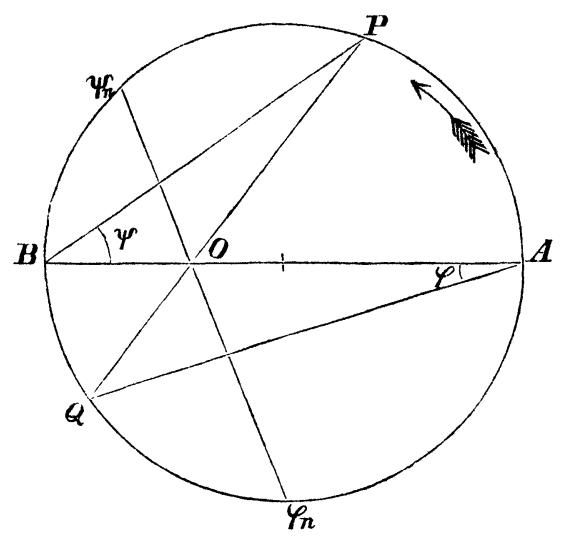

On peut s'en convaincre par une considération géométrique fort simple $\left.{ }^{*}\right)$. Sur le diamètre $A B$ d'un cercle (Fig. 2) soit pris le point fixe $O$, tel que $O A=a, O B=b$. Concevons une droite mobile $P Q$, qui, partant de la position initiale $A B$ tourne indéfinement et toujours dans le même sens sur le point $O$; les points $P, Q$, où elle coupe la circonférence, détermineront deux arcs de cercle $\boldsymbol{A P}, \boldsymbol{B Q}$, ou deux angles $\boldsymbol{P B A}=\psi$, $Q A B=\varphi$. Ces angles varient d'une manière continue en passant par tous les états de grandeur; ils acquièrent ensemble les valeurs $0, \pi, 2 \pi$, quand $P Q$ se confond pour la $1^{\mathrm{e}}, 2^{\text {ième }}, 3^{\text {iène }}$ fois avec $A B$; enfin ils sont liés par la relation:

A l'aide du lemme (1.) on a:

$$
\frac{\operatorname{tang} \psi}{\operatorname{tang} \psi}=\frac{a}{b} \text {. }
$$

Or

$$
\frac{d \psi}{O P}=\frac{d \varphi}{O Q} .
$$

$$
O P=a \sqrt{1-\frac{a^{2}-b^{2}}{a^{2}} \sin ^{2} \psi}=a \Delta \psi ;
$$

d'où par la permutation des lettres $a, b$ :

par suite :

$$
O Q=b \sqrt{1+\frac{a^{2}-b^{2}}{b^{2}} \sin ^{2} \varphi}=b \Delta^{\prime} \varphi ;
$$

$$
\frac{d \psi}{\Delta \psi}=\frac{a}{b} \frac{d \varphi}{\Delta^{\prime} \varphi} .
$$

Ainsi les deux intégrales $\int_{0}^{\psi_{0}} \frac{d \psi}{\Delta \psi}$ et $\int_{0}^{\varphi_{0}} \frac{d \varphi}{\Delta^{\prime} \varphi}$ sont dans le rapport constant $a: b$. Donc, si l'on connait l'amplitude $\psi_{n}$ d'une intégrale $=n \int_{0}^{\psi_{0}} \frac{d \psi}{\Delta \psi}=n u_{1}$ l'angle $\varphi_{n}$, fourni par notre construction, sera l'amplitude d'une intégrale $\int_{0}^{\varphi_{n}} \frac{d \varphi}{d_{9}^{\prime} \varphi}=n \int_{0}^{\varphi} \frac{d \varphi}{\Delta^{\prime} \varphi}$. De plus, il est manifeste, que quand on sera parvenu à former une équation entre $\psi_{n}, \psi_{11}, a, b$, on n'aura qu'à y remplacer $\psi$ par

*) C'est cette considération géométrique dont Jacobi s'est servie pour construire la substitution de'Landen (Lettre à M. Hermite, vol. 32 pag. 178 de ce Journal). 
$\varphi$ et à permuter $a, b$, pour avoir une équation, à laquelle un procédé analogue aurait conduit. Mais comme alors $\Delta \psi$ se change en $\Delta^{\prime} \dot{\varphi}$, on voit, que $\frac{\sin \psi_{n}}{\sin \psi_{0}}$ et $\cos \psi_{n}$ se changent en $\frac{\sin \varphi_{n}}{\sin \varphi_{0}}$ et $\cos \varphi_{n}$, lorsque dans les expressions qui servent à calculer les premières on écrit $\cos \varphi$ au lieu de $c, \frac{\cos \varphi}{\Delta^{\prime} \varphi}$ au lieu de $s$. Or, la figure met en évidence les relations:

$$
\cos \varphi=\frac{\cos \psi}{\Delta \psi}=s, \quad \frac{\cos \varphi}{\Delta^{\prime} \varphi}=\cos \psi=c ;
$$

par conséquent le changement indiqué revient à permuter $s_{0}, c_{v}$; d'ailleurs puisque $\cos \varphi=s=\sin \operatorname{coam} u$, les formules $\cos \varphi_{n}, \frac{\sin \varphi_{n}}{\sin \varphi_{0}}$ représentent la même chose que $\sin \operatorname{coam} n u_{0}, \frac{\cos \operatorname{coam} n u_{0}}{\cos \operatorname{coam} u_{0}}$.

Actuellement, en examinant les quatre formules, qui permettent de calculer les fonctions $\cos \operatorname{am} 2 h u_{0}, \frac{\sin \operatorname{am} 2 h u_{0}}{s_{0} \sin \operatorname{am} u_{0}}, \frac{\cos \operatorname{am}(2 h+1) u_{0}}{c_{0}}, \frac{\sin \operatorname{am}(2 h+1) u_{0}}{\sin \operatorname{am} u_{0}}$, ainsi que celles qui s'en déduisent par la permutation des lettres $s$, $c$, on reconnait facilement, qu'elles ne renferment que des puissances paires de $s_{0}, c_{0}$. En effet, on en serait assuré, si l'on savait que, suivant que $h$ est impair ou pair, les fonctions:

$$
\frac{\cos a m h u_{0}}{c_{0}}, \quad \frac{\sin a m h u_{0}}{\sin \operatorname{am} u_{0}}, \quad \operatorname{cosam}(h+1) u_{0}, \quad \frac{\sin \operatorname{am}(h+1) u_{0}}{s_{0} \sin \operatorname{am} u_{0}},
$$

ou celles-ci :

$$
\operatorname{cosam} h u_{0}, \quad \frac{\sin \operatorname{am} h u_{0}}{s_{0} \sin a \mathrm{~m} u_{0}}, \quad \frac{\cos a m(h+1) u_{0}}{c_{0}}, \quad \frac{\sin \operatorname{am}(h+1) u_{0}}{\sin \operatorname{am} u_{0}}
$$

jouissent de la même propriété.

Or, puisque cela a lieu pour la première catégorie, si $h=1$, il en sera de même de la seconde, où l'on suppose d'abord $h=2$, et puis $h=4$; alors, les fonctions provenant de la première série pour $h=3$ étant comprises parmi celles que fournit la seconde catégorie pour $h=2, h=4$; la même chose se trouve démontrée pour $h=3$. Par là pour $h=6$ et pour le cas $h=5$, lequel est contenu dans les cas $h=4, h=6$; ainsi de suite.

8. Concevons les deux conjuguées de $E$ par rapport aux ang: :s am $u_{0}$ et am $n u_{1}$, et nommons $\sqrt{a^{2}+\lambda_{0}}, \sqrt{b^{2}+\lambda_{0}} ; \sqrt{a^{2}+\lambda}, \sqrt{b^{2}+\lambda}$ leurs demi-axes. D'après ce que nous venous d'exposer, il sera facile, d'exprimer $\lambda$ par $\lambda_{0}$. En effet, qu'on se reporte aux formules (III.), et qu'on les mette sous la forme: 


$$
\begin{gathered}
a^{2}+\lambda=a^{2} \frac{\cos a m n u_{0}+\sin \operatorname{coam} n u_{0}}{\sin \operatorname{coam} n u_{0}}, \\
c_{0}=\frac{a^{2} b^{2}-\lambda_{0}^{2}}{a^{2}\left(b^{2}+\lambda_{0}\right)+\lambda\left(a^{2}+\lambda_{0}\right)}, \quad s_{0}=\frac{a^{2} b^{2}-\lambda_{0}^{2}}{b^{2}\left(a^{2}+\lambda_{0}\right)+\lambda\left(b^{2}+\lambda_{0}\right)},
\end{gathered}
$$

on reconnait qu'à l'aide d'elles on peut obtenir $\lambda$ en fonction rationelle de $c_{0}, s_{0}$ et de $\lambda_{0}$.

Par exemple $n=2$ :

$$
\lambda=\frac{4 a^{2} b^{2} \lambda_{0}\left(a^{2}+\lambda_{0}\right)\left(b^{2}+\lambda_{0}\right)}{\left(a^{2} b^{2}-\lambda_{0}^{2}\right)^{2}}=a^{2} \frac{1-s_{0}^{2}}{s_{0}^{2}} .
$$

Cela découle au reste immédiatement de la liaison géométrique, établie entre $\psi_{0}=\operatorname{am} u_{0}$ et $\psi=\operatorname{am} 2 u_{0}$ par la construction du $n^{0} 6$. Car, de ce que la tangente au point $\psi_{0}$ doit passer par l'extrémité $B$ du demi-axe $\sqrt{b^{2}+\lambda}$, on conclut

$$
c_{0}^{2}=\frac{b^{2}}{b^{2}+\lambda}, \quad s_{0}^{2}=\frac{a^{2}}{a^{2}+\lambda} .
$$

Mais on a de même d'après la formule (III.):

$$
a^{2}+\lambda_{0}=a^{2} \frac{c_{0}+s_{0}}{s_{0}\left(1+c_{0}\right)}
$$

par conséquent

$$
a^{2}+\lambda_{0}=a \frac{a \sqrt{b^{2}+\lambda}+b \sqrt{a^{2}+\lambda}}{b+\sqrt{b^{2}+\lambda}}
$$

ou

$$
\lambda_{0}=\frac{a b}{\lambda}\left(\sqrt{a^{2}+\lambda}-a\right)\left(\sqrt{b^{2}+\lambda}-b\right) .
$$

Faisant $\lambda=\infty$, on en tire $\lambda_{0}=a b$; et on trouve $\sqrt{a^{2}+a b}, \sqrt{b^{2}+a b}$ pour les demi-axes de l'ellipse, qui sert à diviser $E$ en $2^{2}$ parties égales. En remplaçant $\lambda$ par la valeur trouvée, on calculera successivement les axes des conjuguées de $E$ par rapport aux angles $\frac{\omega}{2}, \frac{\omega}{2^{2}}, \ldots \frac{\omega}{2^{n}} \cdot$ Maintenant, si l'on suppose $u_{0}=\frac{\omega}{n}$, on obtient une équation en $\lambda_{0}$, ainsi qu'il suit:

$1^{\circ}$. Soit $n$ un nombre impair $=2 m+1$. Imaginons l'ellipse entière $E$ divisée en $2 m+1$ parties égales, le point 0 étant le point de départ. En ayant égard à ce que la tangente au $m^{\text {iène }}$ point de division doit passer par l'extrémité du demi-axe $\sqrt{b^{2}+\lambda_{0}}$, on voit:

$$
\cos ^{2} \operatorname{am} m u_{v}=\frac{b^{2}}{b^{2}+\lambda_{0}} .
$$

De là on déduit l'équation cherchée en exprimant $\cos a m m \varkappa_{1}$ par $\lambda_{10}$. 
$2^{\circ}$. Soit $n$ le double d'un nombre impair $=2(2 m+1)$. Imaginons, qu'on divise $E$ en $n$ parties égales et qu'on mène au $m^{\text {ième }}$ point de la division une tangente; celle-ci passera par l'extrémité de l'axe $\sqrt{a^{2}+\lambda_{11}}$, et il suit:

ou

$$
\sin ^{2} \operatorname{am} m u_{11}=\frac{a^{2}}{a^{2}+\lambda_{0}}
$$

$$
\cos ^{2} \operatorname{am} m u_{0}=\frac{\lambda_{0}}{a^{2}+\lambda_{0}} .
$$

Ainsi, dans chacun de ces deux cas, on n'aura qu'à former l'expression qui donne cosam $m u_{0}$ en fonction de $\lambda_{0}$. Mais si l'on considère, que $c_{i j}, s_{0}$ ne changent pas de valeur absolue, mais seulement de signe lorsqu'on remplace $\lambda_{0} \operatorname{par} \frac{a^{2} b^{2}}{\lambda_{0}}$, et que, par conséquent, $\cos ^{2} a m m u_{0}$ n'éprouve aucun changement par cette substitution; on voit, que l'équation relative au premier cas se transforme dans celle qui convient au second, quand on substitue $\frac{a^{2} b^{2}}{\lambda_{0}}$ au lieu de $\lambda_{1)}$, et réciproquement.

Exemple: $n=3, m=1$.

$1^{\text {er }}$ cas: $\cos \operatorname{am} m u_{0}=c_{v}=\frac{a^{2} b^{2}-\lambda_{0}^{2}}{a^{2} b^{2}+2 a^{2} \lambda_{0}+\lambda_{0}^{2}}$, donc

d'où :

$$
\left(\frac{a^{2} b^{2}-\lambda_{0}^{2}}{a^{2} b^{2}+2 a^{2} \lambda_{0}+\lambda_{0}^{2}}\right)^{2}=\frac{b^{2}}{b^{2}+\lambda_{0}},
$$

$$
\begin{gathered}
\lambda_{0}^{4}-6 a^{2} b^{2} \lambda_{1}^{2}-4 a^{2} b^{2}\left(a^{2}+b^{2}\right) \lambda_{0}-3 a^{4} b^{4}=0 . \\
2^{\text {ième }} \text { cas : } n=6, m=1 . \quad \text { Remplaçant } \lambda_{0} \text { par } \frac{a^{2} b^{2}}{\lambda_{0}}, \text { on a: } \\
3 \lambda_{0}^{4}+4\left(a^{2}+b^{2}\right) \lambda_{0}^{3}+6 a^{2} b^{2} \lambda_{0}^{2}-a^{4} b^{4}=0 .
\end{gathered}
$$

Par la supposition $a^{2}=2 b^{2}$, laquelle se rapporte à la division de la lemniscate, les premiers membres de ces équations se décomposent en deux facteurs du second degré. L'équatiọn relative au cas $n=3$ devient, en remplaçant $\lambda_{1}$ par $z$ :

$$
\left(z^{2}+2 b^{2} \sqrt{ } 3 z+2 b^{4} / 3\right)\left(z^{2}-2 b^{2} / 3 z-2 b^{4} / 3\right)=0 .
$$

Des deux valeurs réelles de $z$ savoir $(\sqrt{3+2 \sqrt{3}}+\sqrt{3}) b^{2}$ et $(\sqrt{3}-\sqrt{3+2 / 3}) b^{2}$, la première étant prise pour $\lambda_{0}$, fournit les axes de la conjuguée de $E$ par rapport à am $\frac{\omega}{3}$, ensuite $\lambda_{v}=\frac{2 b^{2}}{\sqrt{3+2 \sqrt{3}}+\sqrt{3}}$ donne les axes de la conjuguée de $E$ par rapport à am $\frac{\omega}{6}$.

9. Problème. Étant données les conjuguées de $E$ par rapport aux angles am $\frac{\omega}{n}$, am $\frac{\omega}{m}$ où l'on suppose $m, n$ premiers entre eux, construire la conjuguée de $E$ par rapport à $\frac{\omega}{m n}$. 
Employons les deux conjuguées données, pour diviser $E$ en $m$ et en $n$ parties égales, de sorte que ces divisions partent du même point $o$, lequel est d'ailleurs arbitraire. Soient $0, \psi_{1}, \psi_{2}, \ldots$ les points de la première division, $o, \varphi_{1}, \varphi_{2}, \ldots$ ceux de la seconde; soient $\mu, \nu$ deux nombres entiers, qui satisfont à l'équation $\nu m-\mu n= \pm 1$. En $\psi_{\nu}, \varphi_{\mu}$ menons deux tangentes à $E$, leur point de concours appartiendra à l'ellipse cherchée.

Imaginons, qu'après avoir divisé $\boldsymbol{E}$ en $n$ parties égales en $o, \psi_{1}, \psi_{2}, \ldots$, on parte successivement de chacun de ces points pour la diviser $n$ fois de suite en $m$ parties égales, de cette manière on obtiendra $m n$ points distincts, qui déterminent évidemment la division de $E$ en $m n$ parties égales. Si $m, n$ ne sont pas premiers entre eux, en procédant comme il a été dit, on divisera $E$ en autant de parties égales qu'il y a d'unités dans le plus petit multiple $v$ de $m, n$. Car, posons $m=t x, n=t y$, où $t$ est le plus grand commun diviseur entre $m$ et $n$. Il est aisé de voir, que les $y$ divisions qui ont pour points de départ $o, \psi_{1}, \psi_{2}, \ldots \psi_{y}$ fournissent $y m=v$ points distincts, et qu'en partant de $\psi_{y+1}, \psi_{y+2}, \ldots$ on doit retrouver ces mèmes points dans le mème ordre qu'auparavant. D'ailleurs il est manifeste, que ces $v$ points appartiennent à la division de $E$ en $v$ parties égales. $\operatorname{am} \frac{\omega}{v}$.

Ainsi, les amplitudes de $\frac{\omega}{m}, \frac{\omega}{n}$ étant connues, on saura construire

10. Application à la Lemniscate. Pour appliquer à la lemniscate les résultats obtenus, nous ferons dépendre la construction de cette courbe de celle d'une ellipse aux demi-axes $M B=b, M A=b / 2$ (Fig. 3).

Fig. 3.

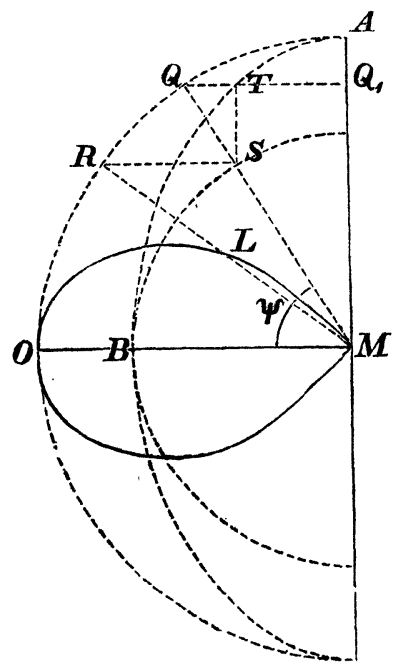

Soit $T$ un point de l'ellipse, déterminé par l'angle $\psi$, de sorte qu'on ait $Q Q_{1}=b / 2 \cos \psi ;$.on trouvera un point $L$, correspondant à $T$ de la manière suivante:

Du centre $M$, avec des rayons $=M A, M B$ nous décrivons deux cercles; par $T$ nous tirons $T S$ perpendiculairement à $M B$, par $S$, où cette perpendiculaire rencontre le petit cercle nous menons à $M B$ une parallèle, qui coupe en $\boldsymbol{R}$ le grand cercle; enfin sur $M R$ nous faisons $M L=Q Q_{1}$. Alors le point $L$ appartient à la lemniscate, et il limite un arc $O L$ de cette courbe, tel que :

$$
\operatorname{arc} O L=b \cdot F\left(\sqrt{1} \frac{1}{2}, \psi\right) \text {. }
$$


L'arc correspondant de l'ellipse a pour mesure:

$$
\operatorname{arc} B T=b / 2 E\left(\gamma_{2}^{1}, \psi\right) \text {. }
$$

Or, pour avoir des arcs de lemniscate égaux, on prendra des arcs d'ellipse égaux, et construira les arcs de lemniscate, dont les extrémités correspondent à celles de ces arcs d'ellipse.

Trèves, 1863.

\section{No te I ̀̀re.}

Les théorèmes 2., 3. établissent des rapports entre deux coniques homofocales, mais on apperçoit que le premier est moins général que le second, en ce qu'il exige que les coniques en question ne se coupent pas, tandisque cette restriction est inutile pour le second. Cependant, en raisonnant comme nous l'avons fait dans la démonstration de 2., on verifiera les énoncés suivants :

$1^{\circ}$. Lorsque par le sommet $P$ d'un angle circonscrit à une ellipse $E$ on fait passer une hyperbole homofocale à $E$ et coupant celle-ci en un point $M$, ce point divisera en deux parties égales l'arc d'ellipse compris entre les côtés de l'angle donné. La différence de deux arcs d'ellipse égaux et contigus est égale à la différence des deux tangentes à leurs extrémités non coincidentes.

En effet, si l'on considère deux angles $S P T, S^{\prime} P^{\prime} T^{\prime}$, circonscrits à $E$, et dont les sommets $P, P^{\prime}$ sont deux points infiniment voisins de l'hyperbole $P M$, on n'a qu'à se rappeler, que la droite $P P^{\prime}$ divise en deux parties égales l'angle $S P T$, pour voir qu'en passant de cet angle à $S^{\prime} P^{\prime} T^{\prime}$, la différence des deux tangentes $S P, T P$ varie exactement comme la différence des arcs $S M$, $T M$ et que ces variations ont pour mesure: $S S^{\prime}-T T^{\prime}$. Et cette observation conduit évidemment à la conclusion:

$$
S P-T P=S M-T M .
$$

$2^{\circ}$. Lorsque par le sommet d'un angle circonscrit à une branche d'hyperbole on fait passer une ellipse, homofocale à cette hyperbole et la coupant en un point $M$, ce point divisera en deux parties égales l'arc d'hyperbole compris entre les côtés de l'angle donné. La différence de deux arcs d'hyperbole égaux et contigus est égale à la différence des deux tangentes à leur extrémités non coincidentes. 
$3^{\circ}$. La même chose pour deux paraboles homofocales qui se coupent.

On tire de là une nouvelle solution du problème 6., savoir:

Pour partager en deux parties égales un arc de conique $T_{1} T_{2}$, on lui circonscrira un angle $T_{1} P T_{2}$, et par $P$ on fera passer une conique homofocale qui coupe l'arc $T_{1} T_{2}$ en $M$ : ce point sera le milieu cherché.

En rapprochant nos deux solutions, on trouve:

Lorsqu'à un cercle variable, qui touche une conique $E$ extérieurement en un point fixe $M$ on circonscrit un angle, de façon qu'il soit de même circonscrit $\grave{a} E$, son sommet sera sur une conique homofocale $\grave{a} E$ et coupant celle-ci en $M$.

On a ce cas particulier: Lorsqu'à un cercle variable, qui touche une droite $m$ en un point fixe $M$ on circonscrit un angle, dont les côtés doivent passer par deux points fixes $F, F^{\prime}$ de $m$, le sommet de cet angle restera sur une conique décrite des foyers $F, F^{\prime}$. On peut remplacer la droite $m$ par un plan $m$, le cercle variable par une sphère variable touchant $m$ au point fixe $M$, l'angle circonscrit par un cône circonscrit à la sphére, lequel a pour trace dans le plan $m$ une conique, dont $M$ est l'un des foyers; alors on aura le théorème connu: Le lieu géométrique des sommets des cônes de révolution qui passent par une conique donnée, est une conique située dans un plan perpendiculaire au plan de la première; chacune de ces deux coniques a pour foyers les extrémités du grand axe de l'autre.

Voici une proposition qu'on démontre facilement en combinant les théorèmes $2 ., 3$. avec $1^{\circ}, 2^{\circ}, 3^{\circ}$ :

Soient $m, n$ les côtés d'un angle variable circonscrit à une conique $E$, et dont le sommet est censé parcourir une conique $E^{\prime}$, homofocale à $E$; soient $m^{\prime}, n^{\prime}$ les côtés d'un angle également circonscrit à $E$, et placé symétriquement au premier par rapport à l'un des axes de $E$, de maniére que les côtés symétriques $m, m^{\prime}$ et $n, n^{\prime}$ se coupent sur cet axe ou sur son prolongement: Alors les points d'intersection des droites $m, n^{\prime}$ et $n, m^{\prime}$ seront situés sur une conique $E^{\prime \prime}$ homofocale à $E$, et ces coniques $E^{\prime \prime}, E$ se couperont, ou ne se couperont pas, selon que $E^{\prime}, E$ ne se coupent pas ou se coupent. 


\section{Note $2^{\text {ième }}$.}

Sur la relation qui existe entre les limites d'intégrales elliptiques de $1^{i e ̀ r e}$ espèce, ayant une valeur constante.

Nommons $\psi_{1}, \psi_{2}$ (Fig. 4) les limites d'une telle intégrale, $T_{1}, T_{2}$ les points de $E$, déterminés par les angles $\psi_{1}, \psi_{2}$ (voyez 4.); la relation dont il s'agit est l'expression analytique de ce fait, que les tangentes en $T_{1}, T_{2}$ se coupent sur l'ellipse $E^{\prime}$, homofocale à $E$. On la trouve rapidement ainsi qu'il suit:

Concevons qu'on incline le plan qui contient $E, E^{\prime}$ vers le plan du cercle décrit avec le demi-axe ${ }^{b}$ $b$ comme rayon, de manière que ce cercle devienne la projection orthogonale de $E: T_{1}^{\prime}, T_{2}^{\prime}, P^{\prime}$ seront les projections de $T_{1}, T_{2}, P$, et le point $P^{\prime}$ appartiendra Fig. 4.

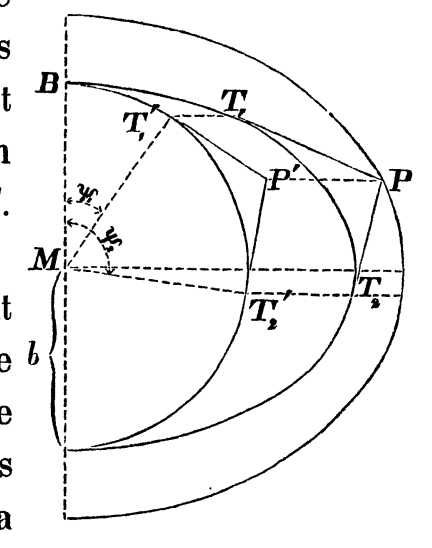
à la projection de $E^{\prime}$, c'est-à-dire à une ellipse aux demi-axes $\sqrt{b^{2}+\lambda}$, $\frac{b}{a} \sqrt{a^{2}+\lambda}$.

Comme on a: angle $B M T_{1}^{\prime}=\psi_{1}, B M T_{2}^{\prime}=\psi_{2}$; et $T_{1}^{\prime} P^{\prime}, T_{2}^{\prime} P^{\prime}$ étant des tangentes du cercle au rayon $b$, on aura:

$$
M P^{\prime}=\frac{b}{\cos \frac{1}{2}\left(\psi_{2}-\psi_{1}\right)} .
$$

Par suite les coordonnées du point $\boldsymbol{P}^{\prime}$ seront:

$$
\frac{b}{\cos \frac{1}{2}\left(\psi_{2}-\psi_{1}\right)} \sin \frac{1}{2}\left(\psi_{1}+\psi_{2}\right) \text { et } \frac{b}{\cos \frac{1}{2}\left(\psi_{2}-\psi_{1}\right)} \cos \frac{1}{2}\left(\psi_{1}+\psi_{2}\right)
$$

Si dans l'équation de la projection de $E^{\prime}$ on introduit ces valeurs, on obtient par une transformation qui se présente d'elle même:

$$
\cos \psi_{1} \cos \psi_{2}+\frac{b^{2}\left(a^{2}+\lambda\right)+\lambda\left(b^{2}+\lambda\right)}{a^{2}\left(b^{2}+\lambda\right)+\lambda\left(a^{2}+\lambda\right)} \sin \psi_{1} \sin \psi_{2}=\frac{a^{2}\left(b^{2}+\lambda\right)-\lambda\left(a^{2}+\lambda\right)}{a^{2}\left(b^{2}+\lambda\right)+\lambda\left(a^{2}+\lambda\right)} .
$$

Posons $\psi_{1}=0, \psi_{2}=\psi$, il vient:

$$
\cos \psi=\frac{a^{2}\left(b^{2}+\lambda\right)-\lambda\left(a^{2}+\lambda\right)}{a^{2}\left(b^{2}+\lambda\right)+\lambda\left(a^{2}+\lambda\right)} ; \text { de là : } \Delta \psi=\frac{b^{2}\left(a^{2}+\lambda\right)+\lambda\left(b^{2}+\lambda\right)}{a^{2}\left(b^{2}+\lambda\right)+\lambda\left(a^{2}+\lambda\right)} .
$$

Ces équations permettent d'exprimer $a^{2}+\lambda, b^{2}+\lambda$ en fonctions de $\psi$, et de mettre la relation trouvée sous sa forme ordinaire:

$$
\cos \psi_{1} \cos \psi_{2}+\sin \psi_{1} \sin \psi_{2} \Delta \psi=\cos \psi
$$

